\title{
EVENTUAL DISCONJUGACY OF A LINEAR DIFFERENTIAL EQUATION. II
}

\author{
WILLIAM F. TRENCH
}

ABSTRACT. A sufficient condition is given for eventual disconjugacy of an $n$ th-order linear differential equation. The condition is weaker than one given in an earlier paper with the same title, and the conclusion is sharper.

We consider the equation

$$
y^{(n)}+p_{1}(t) y^{(n-1)}+\cdots+p_{n}(t) y=0, \quad t \geqslant a,
$$

where $p_{1}, \ldots, p_{n}$ are real-valued and continuous on $[a, \infty)$ and $n \geqslant 2$. If there is an $a_{1} \geqslant a$ such that no nontrivial solution of (1) has more than $n-1$ zeros (counting multiplicities) on $\left[a_{1}, \infty\right)$, then (1) is said to be eventually disconjugate. It is known [2] that (1) is eventually disconjugate if it has a fundamental system $y_{0}, \ldots, y_{n-1}$ such that

(2)

$y_{m}^{(r)}(t)=\left\{\begin{array}{ll}t^{m-r}(1+o(1)) /(m-r) !, & 0 \leqslant r \leqslant m, \\ o\left(t^{m-r}\right), & m+1 \leqslant r \leqslant n-1,\end{array} \quad 0 \leqslant m \leqslant n-1\right.$.

(Throughout this paper " $O$ " and " $O$ " refer to behavior as $t \rightarrow \infty$.)

By a theorem of Hartman and Wintner [1] such a fundamental system exists if

$$
\int^{\infty} t^{k-1}\left|p_{k}(t)\right| d t<\infty, \quad \therefore 1 \leqslant k \leqslant n \text {. }
$$

Consistent with this, a theorem of Willett [4] also implies that (3) is a sufficient condition for eventual disconjugacy of (1). We proved the following theorem in [3].

THEOREM 1. If the integrals

$$
\int^{\infty} t^{k-1} p_{k}(t) d t, \quad 1 \leqslant k \leqslant n,
$$

converge ( perhaps conditionally for $2 \leqslant k \leqslant n$ ),

$$
\int^{\infty}\left|p_{1}(t)\right| d t<\infty
$$

Received by the editors June 6, 1984. Presented to the Society, January, 1985. 1980 Mathematics Subject Classification. Primary 34C10.

c)1985 American Mathematical Society $0002-9939 / 85 \$ 1.00+\$ .25$ per page 
and

$$
\int^{\infty}\left|\int_{t}^{\infty}(t-s)^{k-2} p_{k}(s) d s\right| d t<\infty, \quad 2 \leqslant k \leqslant n,
$$

then (1) has a fundamental system $y_{0}, \ldots, y_{n-1}$ which satisfies (2), and therefore (1) is eventually disconjugate.

It was shown in [3] that (4)-(6) are weaker than (3).

Obviously, the convergence of the integrals in (4) implies that

$$
\lim _{t \rightarrow \infty} \int_{t}^{\infty} s^{k-1} p_{k}(s) d s=0, \quad 1 \leqslant k \leqslant n .
$$

Here we show that this trivial observation can be exploited to weaken assumptions (5) and (6) and even eliminate the latter altogether in some cases.

The following lemma from [3] facilitates the statement of our main theorem and is used in its proof.

LemMa 1. Suppose $u \in C[0, \infty)$ and $\int^{\infty} t^{k-1} u(t) d t$ converges ( perhaps conditionally) for some $k \geqslant 1$. Define

$$
I_{0}(t ; u)=u(t)
$$

and

$$
I_{j}(t ; u)=\int_{t}^{\infty} I_{j-1}(s ; u) d s=\int_{t}^{\infty} \frac{(s-t)^{j-1}}{(j-1) !} u(s) d s, \quad 1 \leqslant j \leqslant k .
$$

Then the integrals $(8)$ converge and

$$
\left|I_{j}(t ; u)\right| \leqslant 2 \delta(t) t^{j-k} /(j-1) !, \quad 1 \leqslant j \leqslant k
$$

where

$$
\delta(t)=\sup _{\tau \geqslant t}\left|\int_{\tau}^{\infty} s^{k-1} u(s) d s\right| .
$$

Definition 1. Let $\Phi$ be the class of positive, nonincreasing, and continuous functions $\phi$ on $[a, \infty)$ such that

$$
\lim _{t \rightarrow \infty} \phi(t)=0
$$

For each $\phi$ in $\Phi$ define

$$
\hat{\phi}(t)=\frac{1}{t} \int_{a}^{t} \phi(s) d s
$$

and

$$
\phi_{M}=\max \{\hat{\phi}, \phi\}
$$

It is easy to show that

$$
\lim _{t \rightarrow \infty} \hat{\phi}(t)=\lim _{t \rightarrow \infty} \phi_{M}(t)=0 .
$$

The following is our main theorem.

THEOREM 2. Suppose the integrals (4) converge and there is a function $\phi$ in $\Phi$ such that

$$
\int_{t}^{\infty} s^{k-1} p_{k}(s) d s=O(\phi(t)), \quad 1 \leqslant k \leqslant n
$$


and

$$
\varlimsup_{t \rightarrow \infty}(\phi(t))^{-1} \int_{t}^{\infty}\left|I_{k-1}\left(s ; p_{k}\right)\right| \phi(s) d s=\theta_{k}, \quad 1 \leqslant k \leqslant n,
$$

where $\theta_{1}, \ldots, \theta_{n}$ are sufficiently small. Then (1) has a fundamental system $y_{0}, \ldots, y_{n-1}$ such that

$$
y_{m}^{(r)}(t)= \begin{cases}t^{m-r}(1+O(\hat{\phi}(t)) /(m-r) !, & 0 \leqslant r \leqslant m-1 \\ 1+O(\phi(t)), & r=m, \\ O\left(\phi(t) t^{m-r}\right), & m+1 \leqslant r \leqslant n-1\end{cases}
$$

$(0 \leqslant m \leqslant n-1)$, and therefore (1) is eventually disconjugate.

Some comments are in order before we prove Theorem 2. Its hypotheses are weaker than those of Theorem 1, since (7) obviously implies (15) for some $\phi$ in $\Phi$; for example, if

$$
\delta_{k}(t)=\sup _{\tau \geqslant t}\left|\int_{\tau}^{\infty} s^{k-1} p_{k}(s) d s\right|, \quad 1 \leqslant k \leqslant n,
$$

then the function $\phi=\max \left\{\delta_{1}, \ldots, \delta_{n}\right\}$ is in $\Phi$ and (15) holds. Moreover, (5) and (6) imply (16) with $\theta_{k}=0$ for every $\phi$ in $\Phi$, while (16) may hold for some $\phi$ in $\Phi$ even though one or both of (5) and (6) fail to hold. Finally, (17) is obviously sharper than (2).

Corollary 1. Suppose (15) holds for some $\phi$ in $\Phi$ such that

$$
\varlimsup_{t \rightarrow \infty}(\phi(t))^{-1} \int_{t}^{\infty} \frac{\phi^{2}(s)}{s} d s=\alpha<\infty,
$$

and let

$$
\beta_{k}=\varlimsup_{t \rightarrow \infty}(\phi(t))^{-1} \delta_{k}(t), \quad 2 \leqslant k \leqslant n .
$$

Suppose also that

$$
\varlimsup_{t \rightarrow \infty}(\phi(t))^{-1} \int_{t}^{\infty} \phi(s)\left|p_{1}(s)\right| d s=\theta_{1} .
$$

Then the conclusions of Theorem 1 hold if $\alpha \beta_{2}, \ldots, \alpha \beta_{k}$, and $\theta_{1}$ are all sufficiently small.

Proof. For $k=1,(16)$ and (21) are the same. For $2 \leqslant k \leqslant n$, Lemma 1 (specifically, (9) with $u=p_{k}$ ) implies that

$$
\left|I_{k-1}\left(s ; p_{k}\right)\right| \leqslant 2 \delta_{k}(s) s^{-1} /(k-2) ! .
$$

Therefore, (19) and (20) imply (16), with

$$
\theta_{k} \leqslant 2 \alpha \beta_{k} /(k-2) !, \quad 2 \leqslant k \leqslant n .
$$

Condition (19) holds, for example, with $\alpha=0$ if $\phi(t)=t^{-a}$ for some $a>0$, or if $\phi(t)=(\log t)^{-a}$ with $a>1$; it also holds with $\alpha=1$ if $\phi(t)=(\log t)^{-1}$.

The next lemma will be used to prove Theorem 2 .

LemMA 2. Suppose $\phi \in \Phi, t_{0}>a$, and $m$ is an integer, $0 \leqslant m \leqslant n-1$. Let $B_{m}\left(t_{0}\right)$ be the Banach space of functions $h$ in $C^{(n-1)}\left[t_{0}, \infty\right)$ such that

$$
h^{(r)}(t)= \begin{cases}O\left(\hat{\phi}(t) t^{m-r}\right), & 0 \leqslant r \leqslant m-1, \\ O\left(\phi(t) t^{m-r}\right), & m \leqslant r \leqslant n-1,\end{cases}
$$


with norm

$$
\|h\|=\sup _{t \geqslant t_{0}}\left\{(\hat{\phi}(t))^{-1} \sum_{r=0}^{m-1} t^{r-m}\left|h^{(r)}(t)\right|+(\phi(t))^{-1} \sum_{r=m}^{n-1} t^{r-m}\left|h^{(r)}(t)\right|\right\} .
$$

Suppose $u \in C\left[t_{0}, \infty\right)$ and

$$
\int_{t}^{\infty} s^{n-m-1} u(s) d s=O(\phi(t)),
$$

where the integral may converge conditionally, and define

$$
L_{m}(t ; u)= \begin{cases}\int_{t}^{\infty} \frac{(t-s)^{n-1}}{(n-1) !} u(s) d s & \text { if } m=0, \\ \int_{t_{0}}^{t} \frac{(t-\lambda)^{m-1}}{(m-1) !} d \lambda \int_{\lambda}^{\infty} \frac{(\lambda-s)^{n-m-1}}{(n-m-1) !} u(s) d s & \text { if } 1 \leqslant m \leqslant n-1 .\end{cases}
$$

Then

$$
L_{m}(\cdot ; u) \in B_{m}\left(t_{0}\right)
$$

and

$$
\left\|L_{m}(\cdot ; u)\right\| \leqslant K_{m} \sigma\left(t_{0}\right)
$$

where $K_{m}$ is a constant which does not depend on $t_{0}$ or $u$, and

$$
\sigma(t)=\sup _{\tau \geqslant t}\left\{(\phi(\tau))^{-1}\left|\int_{\tau}^{\infty} s^{n-m-1} u(s) d s\right|\right\} .
$$

Moreover,

$$
L_{m}^{(n)}(t ; u)=-u(t) .
$$

Proof. Lemma 1 and (24) imply that the improper integral in the definition of $L_{m}(t ; u)$ converges, and that the improper integrals in the derivatives

$$
L_{m}^{(r)}(t ; u)=\int_{t}^{\infty} \frac{(t-s)^{n-r-1}}{(n-r-1) !} u(s) d s, \quad m \leqslant r \leqslant n-1,
$$

also converge. Moreover, if $\delta$ is as in (10) (with $k=n-m$ ) and $\sigma$ is as in (28), then $\delta(t) \leqslant \sigma(t) \phi(t)$. This, (9), and (30) imply that

$$
\left|L_{m}^{(r)}(t ; u)\right| \leqslant 2 \sigma(t) \phi(t) t^{m-r} /(n-r-1) !, \quad m \leqslant r \leqslant n-1 .
$$

If $m \geqslant 1$, then (25) and (30) (with $r=m$ ) imply that

$$
L_{m}^{(r)}(t, u)=\int_{t_{0}}^{t} \frac{(t-\lambda)^{m-r-1}}{(m-r-1) !} L_{m}^{(m)}(\lambda) d \lambda, \quad 0 \leqslant r \leqslant m-1 .
$$

This and (31) with $r=m$ imply that

$$
\left|L_{m}^{(r)}(t ; u)\right| \leqslant \frac{2}{(n-m-1) !(m-r-1) !} \int_{t_{0}}^{t}(t-\lambda)^{m-r-1} \sigma(\lambda) \phi(\lambda) d \lambda,
$$

and therefore, from (12) and the monotonicity of $\sigma$,

$$
\left|L_{m}^{(r)}(t ; u)\right| \leqslant \frac{2 \sigma\left(t_{0}\right) \hat{\phi}(t) t^{m-r}}{(n-m-1) !(m-r-1) !}, \quad 0 \leqslant r \leqslant m-1 .
$$


Now (31), (32), and the monotonicity of $\sigma$ imply (26) and (27) for some constant $K_{m}$. Since (29) is obvious, the proof is complete.

Proof of Theorem 1 . Let $m$ be fixed, $0 \leqslant m \leqslant n-1$. Define

$$
M h=\sum_{k=1}^{n} p_{k} h^{(n-k)}
$$

and

$$
f_{m}(t)=\sum_{k=n-m}^{n} p_{k}(t) \frac{t^{m-n+k}}{(m-n+k) !} .
$$

Because of (15),

$$
\int_{t}^{\infty} s^{n-m-1} f_{m}(s) d s=O(\phi(t))
$$

We will show that the transformation $\hat{h}=T_{m} h$ defined by

$$
\hat{h}(t)=L_{m}\left(t ; f_{m}\right)+L_{m}(t ; M h), \quad t \geqslant t_{0},
$$

maps $B_{m}\left(t_{0}\right)$ into itself and is a contraction mapping if $t_{0}$ is sufficiently large. It will then follow easily that if $h_{m}$ is the function in $B_{m}\left(t_{0}\right)$ left fixed by $T_{m}$ and

$$
y_{m}(t)=t^{m} / m !+h_{m}(t)
$$

then $y_{m}$ satisfies (1) and (16).

Lemma 2 and (35) imply that $L_{m}\left(\cdot ; f_{m}\right) \in B_{m}\left(t_{0}\right)$. To show that

$$
L_{m}(\cdot ; M h) \in B_{m}\left(t_{0}\right) \quad \text { if } h \in B_{m}\left(t_{0}\right),
$$

it suffices, again by Lemma 2 , to show that the integral

$$
J(t ; M h)=\int_{t}^{\infty} s^{n-m-1}(M h)(s) d s
$$

converges and is $O(\phi(t))$. To this end we follow a procedure used in [3], with appropriate modifications. Using (8) and repeated integration by parts yields

$$
\begin{aligned}
\int_{t}^{i} s^{n-m-1}(M h)(s) d s= & \sum_{k=1}^{n} \int_{t}^{i} s^{n-m-1} p_{k}(s) h^{(n-k)}(s) d s \\
= & -\left.\sum_{k=2}^{n} \sum_{j=1}^{k-1} I_{j}\left(s ; p_{k}\right)\left[s^{n-m-1} h^{(n-k)}(s)\right]^{(j-1)}\right|_{t} ^{i} \\
& +\int_{t}^{i}\left(\sum_{k=1}^{n} I_{k-1}\left(s ; p_{k}\right)\left[s^{n-m-1} h^{(n-k)}(s)\right]^{(k-1)}\right) d s .
\end{aligned}
$$

Because of (22) and (23), there are constants $\left\{b_{j k}\right\}$ which do not depend on $t_{0}$ or $h$, such that

(39)

$$
\left|\left[s^{n-m-1} h^{(n-k)}(s)\right]^{(j-1)}\right| \leqslant b_{j k}\|h\| s^{k-j} \phi_{M}(s), \quad 1 \leqslant j \leqslant k-1 \leqslant n-1, s \geqslant t_{0}
$$

(recall (13)), and

$$
\left|\left[s^{n-m-1} h^{(n-k)}(s)\right]^{(k-1)}\right| \leqslant b_{k k}\|h\| \phi(s), \quad 1 \leqslant k \leqslant n, s \geqslant t_{0} .
$$


It is important to notice that $\phi(s)$ appears on the right of $(40)$, rather than $\phi_{M}(s)$, as in (39). This is because of $(22)$ and the fact that only the derivatives $h^{(m)}, \ldots, h^{(n-1)}$ actually occur on the left of (40), which can be verified by using Leibniz's formula for the derivatives of a product and noting that $\left(s^{n-m-1}\right)^{(\nu)}=0$ if $\nu>n-m-1$.

From (15) and (18),

$$
\delta_{k}(t) \leqslant \lambda_{k} \phi(t), \quad 1 \leqslant k \leqslant n, t \geqslant a,
$$

for some constants $\lambda_{1}, \ldots, \lambda_{n}$. This, (9) with $u=p_{k}$, and (39) imply that

$$
\begin{aligned}
\left|I_{j}\left(s ; p_{k}\right)\left[s^{n-m-1} h^{(n-k)}(s)\right]^{(j-1)}\right| \leqslant \frac{2 b_{j k} \lambda_{k}\|h\|}{(j-1) !} \phi_{M}(s) \phi(s), & 1 \leqslant j \leqslant k-1 \leqslant n-1, s \geqslant t_{0} .
\end{aligned}
$$

Now (16), (40), and (41) imply that we can let $\bar{t} \rightarrow \infty$ in (38) to conclude that $J(t ; M h)$ converges for $t \geqslant t_{0}$ and

$$
|J(t ; M h)| \leqslant\|h\| \rho(t) \phi(t),
$$

where

$$
\begin{aligned}
\rho(t)= & 2\left(\sum_{k=2}^{n} \sum_{j=1}^{k-1} \frac{b_{j k} \lambda_{k}}{(j-1) !}\right) \phi_{M}(t) \\
& +(\phi(t))^{-1} \sum_{k=1}^{n} b_{k k} \int_{t}^{\infty} \phi(s)\left|I_{k-1}\left(s ; p_{k}\right)\right| d s .
\end{aligned}
$$

Hence, $J(t ; M h)=O(\phi(t))$, and Lemma 2 implies (37); moreover, (27) with $u=M h$ and $\sigma=\|h\| \rho$ imply that

$$
\left\|L_{m}(\cdot ; M h)\right\| \leqslant K_{m} \rho\left(t_{0}\right)\|h\| .
$$

From (14), (16), and (42),

$$
\varlimsup_{t \rightarrow \infty} \rho(t) \leqslant \sum_{k=1}^{n} b_{k k} \theta_{k}<\frac{1}{K_{m}},
$$

where the second inequality holds if $\theta_{1}, \ldots, \theta_{n}$ are sufficiently small; therefore, $T_{m}$ is a contraction mapping if $t_{0}$ is chosen so that $K_{m} \rho\left(t_{0}\right)<1$. Then there is an $h_{m}$ in $B_{m}\left(t_{0}\right)$ such that $h_{m}=T_{m} h_{m}$; i.e.,

$$
h_{m}(t)=L_{m}\left(t ; f_{m}\right)+L_{m}\left(t ; M h_{m}\right) \text {. }
$$

Differentiating this and recalling (29) yield

$$
h_{m}^{(n)}=-f_{m}-M h_{m},
$$

which, with the definitions (33) and (34), implies that $y_{m}$ as defined in (36) satisfies (1) on $\left[t_{0}, \infty\right)$ (and can be continued as a solution of (1) over $[a, \infty)$ ). Since $h_{m} \in B_{m}\left(t_{0}\right),(22)$ and (36) imply (17). Since (11), (14), and (17) imply (2), which is a sufficient condition for eventual disconjugacy, this completes the proof. 


\section{REFERENCES}

1. P. Hartman and A. Wintner, Asymptotic integration of linear differential equations, Amer. J. Math. 77 (1955), 45-87.

2. W. F. Trench, A sufficient condition for eventual disconjugacy, Proc. Amer. Math. Soc. 52 (1975), 139-146.

3. Eventual disconjugacy of a linear differential equation, Proc. Amer. Math. Soc. 89 (1983), 461-466.

4. D. Willett, Disconjugacy tests for singular linear differential equations, SIAM J. Math. Anal. 2 (1971), 536-545.

Department of Mathematics and Computer Science, Drexel University, Philadelphia, PennSYLVANIA 19104 